Physics, Chemistry, and Dynamics of Interplanetary Dust

ASP Conference Series, Vol. 104, 1996

Bo A. S. Gustafson and Martha S. Hanner (eds.)

\title{
Mineralogical Changes in IDPS Resulting From Atmospheric Entry Heating
}

\author{
Lindsay P Keller \\ MVA, Inc., 5500 Oakbrook Parkway, Suite 200, Norcross, GA 30093 \\ Kathie L. Thomas \\ Code C23, Lockheed-Martin NASA/JSC, Houston, TX 77058 \\ David S. McKay \\ Code SN, NASA-Johnson Space Center, Houston, TX 77058
}

\begin{abstract}
All extraterrestrial particles are heated during their passage through the Earth's atmosphere. The mineral assemblages that form during entry heating can be used to constrain the maximum temperature attained which, in turn, places limits on their entry velocity. Entry velocity can be used to discriminate typical cometary IDPs from asteroidal particles.

A common feature of heated IDPs is the presence of magnetite (Mt) rims on the particle surface. These rims range from thin, discontinuous Mt lavers on lobes or on small constituent grains within lightly heated IDPs, to thick, continuous, polycrystalline rims that completely surround many strongiy heated particles. Petrographic evidence indicates that Mt rims form directly from individual phases in IDPs via oxidation of $\mathrm{Fe}$ from the host phases. Our data indicate that the temperature of magnetite formation can range from a minirnum of $\sim 600^{\circ} \mathrm{C}$ (by decarbonation of Fe-bearing carbonates and the breakdown of phyllosilicates) to $\mathrm{T}>850^{\circ} \mathrm{C}$ (decomposition of laihunite). In extreme cases, $\mu \mathrm{m}$-sized Mt plates armor the surface of partly melted chondritic IDPs. Magnetite rims are more well-developed on hydrated IDPs than on anhydrous particles. The development of magnetite rims correlates with other independent indicators of heating including loss of volatile trace elements and sulfur depletions.
\end{abstract}

\section{Introduction}

Interplanetary dust particles (IDPs) are pulse-heated as they decelerate in the Earth's atmosphere during entry. The severity of the heating pulse is a complex function of the physical properties and trajectory of the particle (e.g., size, density, encounter velocity, and incidence angle). Theoretical calculations have placed constraints on the peak temperature of the thermal pulse experienced by typical IDPs (e.g. Flynn, 1989; Love and Brownlee, 1994). Both Flynn (1989) and Love and Brownlee (1994) noted that large differences in peak temperatures (up to $300^{\circ} \mathrm{C}$ ) are calculated for typical asteroidal IDPs versus typical cometary particles with identical densities and sizes. Most asteroidal particles enter the atmosphere with velocities of $\sim 12 \mathrm{~km} / \mathrm{s}$ while typical cometary particles enter at speeds $>14 \mathrm{~km} / \mathrm{s}$ (Jackson and Zook, 1992). Understanding the heating effects in IDPs that result from these thermal pulses is important in 
identifying particles derived from objects in asteroidal orbits as opposed to those in cometary orbits.

There are several approaches to the study of atmospheric entry heating including the measurement of volatile trace element abundances (Flynn et al., 1992), release temperatures of noble gases (Nier and Schulter, 1993; Brownlee et al., 1995), solar flare track erasure temperatures (Sandford and Bradley, 1989) and mineralogical analyses (Fraundorf, 1981, Germani et al., 1990; Rietmeijer, 1992; Keller et al., 1992). In this work, we use the mineral assemblages in heated IDPs to constrain the maximum temperature that certain IDPs experienced during atmospheric entry.

\section{Methods}

Individual IDPs were embedded in low viscosity epoxy, and thin sections ( 80 - to 100 -nm thick) were prepared by diamond knife ultramicrotomy IDP thin sections were studied in the transmission electron microscope by highresolution imaging, electron diffraction, and energy-dispersive $\mathrm{x}$-ray spectrometry Bulk chemical compositions for some of the particles were obtained in a scanning electron microscope following the methods of Thomas et al. (1993).

\section{Results and Discussion}

IDPs display a range of mineralogical changes in response to processes such as oxidation, decarbonation, and dehydration that occur during atmospheric entry heating.

Magnetite rims. A typical feature of heated IDPs is the presence of magnetite $\left(\mathrm{Fe}_{3} \mathrm{O}_{4}\right)$ rims on the particle surface (Figure 1). These rims range from thin ( $<50 \mathrm{~nm}$ thick) and discontinuous rims with magnetite grains on lobes or on small constituent grains within the IDP, to thick (up to $\sim 1-2 \mu \mathrm{m}$ thick), continuous, polycrystalline rims that completely surround the particle. We have used the extent of the development of magnetite rims as a relative indicator of the severity of heating. For example, lightly heated particles show no magnetite rims or only poorly-developed, discontinuous rims and the most strongly heated IDPs have the thickest rims (Thomas et al., 1992; Keller et al., 1992, 1993). Many of the most strongly heated particles (i.e. those that have experienced incipient melting) have surfaces that are armoured with large ( $\mu \mathrm{m}$-sized) polycrystalline plates of magnetite (Keller et al., 1992). The development of magnetite rims correlates with other independent indicators of heating including loss of volatile trace elements (particularly Zn, Flynn et al., 1992) and sulfur volatilization (see Thomas et al., this volume).

Petrographic evidence indicates that some discontinuous magnetite rims form directly from individual phases in IDPs via oxidation of Fe from the host phases such as Fe-Ni sulfides, FeNi metal, olivine, phyllosilicates, and Mg-Fe carbonates. In rare instances, there is no clear relationship between the substrate phase and the magnetite rim, for example, magnetite occurs on Fe-free silica glass in IDP L2006A33 and on carbonaceous material in IDP L2005-31-1. In these cases, it appears that Fe has some mobility at the IDP surface during entry heating.

It has been suggested that magnetite rims are condensates that form in the Earth's upper atmosphere from material evaporated from meteoroids (Maurette et al., 1992), however, there are a number of problems with this 
hypothesis. First, the development of magnetite rims correlates with other mineralogical and chemical indicators of strong heating. Second, gas densities are too low and residence times too short to condense the thickness of magnetite observed on some IDPs. Third, it is not clear how a condensation process could deposit a monomineralic layer of magnetite without incorporating other phases (e.g. silicates). And finally, aluminum oxide particles (rocket exhaust) do not show the development of magnetite rims although their residence time in the stratosphere is on a comparable time scale as IDPs.

Degradation of Phyllosilicate Crystallinity. Hydrated IDPs consist largely of fibrous, Fe-bearing phyllosilicates (hydrated sheet-silicates), typically saponite or serpentine. Both types of phyllosilicates have limited thermal stabilities. Experiments show that saponite and serpentine partly dehydrate and become amorphous at $\sim 800^{\circ} \mathrm{C}$ and $\sim 500^{\circ} \mathrm{C}$ respectively (Akai, 1992). Heating to higher temperatures $\left(\sim 900-1000^{\circ} \mathrm{C}\right)$ results in recrystallization to fine-grained olivine and pyroxene. Many hydrated IDPs (especially those $>10 \mu \mathrm{m}$ in dia.) show textural evidence of having undergone strong heating such as alteration to magnetite, a loss of crystallinity, and recrystallization. For example, L2005P9 and U2015C4 are very strongly heated, hydrated IDPs (e.g. both have thick continuous magnetite rims) where the fibrous phyllosilicates have been replaced by amorphous Mg-rich silicate with finely dispersed magnetite grains (Thomas et al. 1992; Ke'ler et al., 1992). In addition to the mineralogical changes, L2005P9 also is highly depleted in volatile trace elements that were lost during entry (Thomas et al., 1992). Interestingly, some hydrated particles with thin discontinuous magnetite rims have phyllosilicates with good crystallinity within a few 10's of nm of the rim (for example, L2006J14). This texture indicates either a low temperature for the onset of Mt rim formation, or an exceedingly short thermal pulse to high temperature.

Decarbonation reactions. A lower limit on the temperature at which magnetite rims begin to form can be obtained from mineral assemblages in certain hydrated IDPs. For example, magnesite-siderite $\left(\mathrm{MgCO}_{3}-\mathrm{FeCO}_{3}\right)$ solid solutions evolve $\mathrm{CO}_{2}$ on heating in air at $\sim 600^{\circ} \mathrm{C}$, and two IDPs (L2006F10, and L2005Q1) contain these carbonates which have partly altered to magnetite. L2005Q 1 also contains solar flare tracks, suggesting that magnetite forms at lower temperatures than the track erasure temperature estimated by Sandford and Bradley (1989).

Laihunite formation. Laihunite $\left[\mathrm{Fe}^{2+}{ }_{(2-3 x)} \mathrm{Fe}^{3+}{ }_{(2 x)} \mathrm{SiO}_{4}\right.$, where $\left.\mathrm{x}=\sim 0.3\right)$ has been identified in two IDPs to date (Keller et al., 1992; Reitmeijer, 1992). Laihunite occurs in terrestrial rocks as a moderate temperature (between 400 and $850^{\circ} \mathrm{C}$ ) oxidation product of $\mathrm{Fe}$-bearing olivine. Laihunite is structurally similar to olivine, and it forms topotactic, lamellar intergrowths within the olivine host. Laihunite occurs in Fe-bearing olivines in a chondritic-porous anhydrous IDP (L2005Q8). In this IDP, the cores of olivine grains are free of laihunite whereas the margins of grains contain a high density of laihunite lamellae. On the outer surface of the IDP, laihunite is partly converted to magnetite. Laihunite has a limited thermal stability and decomposes at $\mathrm{T}>850^{\circ} \mathrm{C}$ to a mixture of magnetite and silica.

\section{Conclusions}

The most common mineralogical effect of atmospheric entry heating is the development of magnetite rims on the outer surfaces of IDPs. Magnetite is stable over a wide temperature range, but can begin to form at peak temperatures 